\title{
Some Applications of Hyperboloid of One Sheet and How to Make the Equation of Helix-Spiral of It
}

\author{
*Manh Hong Do \\ *Hanoi University of Science and Technology, No. 1, Dai Co Viet street, Hai Ba Trung district, Hanoi, Viet Nam \\ School of mechanic, Descriptive geometry and Engineering drawing Department
}

\begin{abstract}
Curves with equations (EQT) are widely used to create surfaces of machine parts in machine manufacture. They make beautiful surfaces that appeal to consumers. These surfaces may be free surfaces or may be assembled surfaces. Helix and spiral curves are very popular applications in mechanical engineering and architecture. They not only are the basis for creating threaded surfaces on machine parts but also create beautiful spiral staircases. This paper not only presents how to make an equations and projections of the Hyperboloid spiral, but also shows its practical applications.
\end{abstract}

Keywords: Equations: EQT, Hyperboloid sprial: Hys, Hyperboloid of one sheet: Hyos.

\section{INTRODUCTION}

Curved surfaces and curves are widely used in engineering. They create very beautiful images on the surface of machine parts and architectural works. Among them is the one-sided hyperboloit. Humans have applied a single-sided hyperboloit to create lots of beautiful towers and structures. Spiral lines on curved surfaces are also very common in engineering. Cylindrical spirals and conical helixes for creating thread surfaces on machine parts. The spiral is also used to create beautiful spiral stairs. The Hys is applied to create soft curves in amusement parks. The following are some applications of the Hyos in shaping devices and structures in the world.

\section{II.MAKE EQUATION OF THE HYPERBOLOID SPRIAL}

\subsection{A geometry Math}

Given a point $\mathrm{M}$ moving at constant speed on straight line 1. The line 1 rotates around the line $t$ with a constant angular velocity. The result will produce 3 following cases.

Case 1: If line $t$ is parallel to line 1 , the trajectory of point $M$ will be a cylindrical spiral.

Case 2: If line $t$ intersects line 1 , then the trajectory of point $\mathrm{M}$ will be a helical cone.
Case 3: If the two lines $t$ and 1 are crossed, the Trajectory of the point $\mathrm{M}$ will be a Hys.

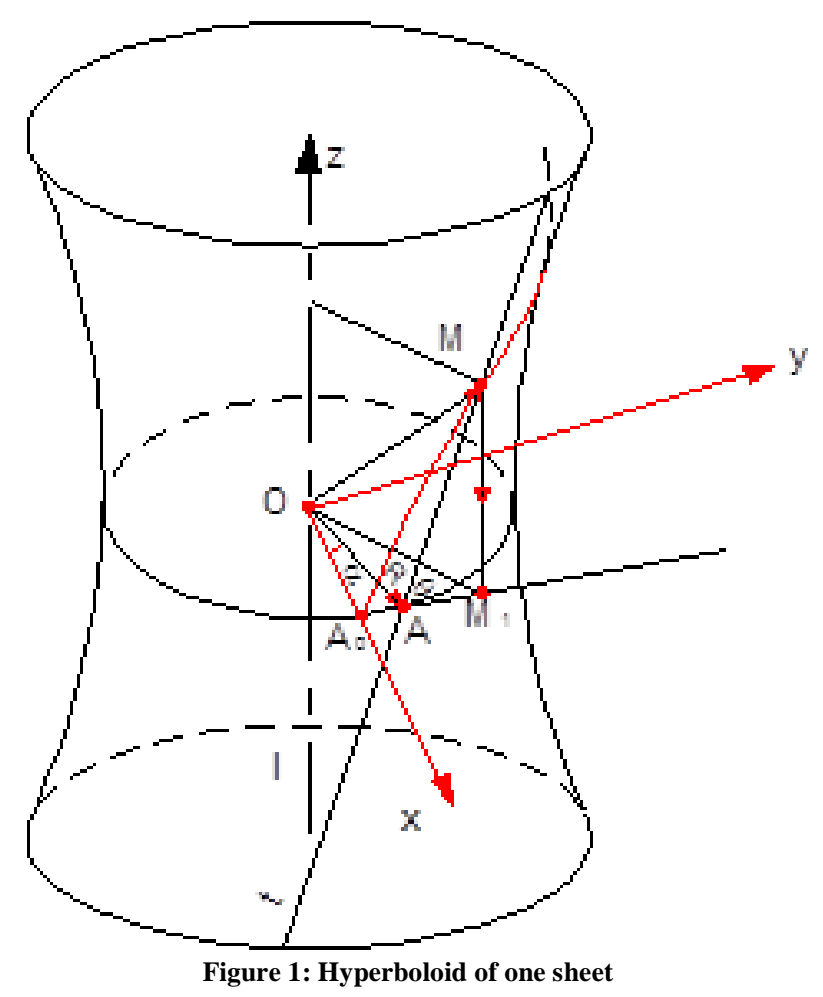

We have: $\mathrm{OA}=\mathrm{R}$

Line OA not only is a radius of the smallest circle but also perpendicular to both line $t$ and line 1 .

$\alpha$ is rotation angle.

When $\alpha=0$ then the first position of point $M$ is the position of point Ao. Make a coordinate system oxyz: $\mathrm{Ox} \equiv \mathrm{OAo}, \mathrm{Oz} \equiv \mathrm{l}$

$\theta$ is the angle between line $t$ and plane xoy.

When $\alpha \neq 0$ then point M move in line t.

We have: $\mathrm{AM}=\mathrm{k} \alpha, \mathrm{k}=$ const.

$$
\overrightarrow{O M}=\overrightarrow{O A}+\overrightarrow{A M}
$$




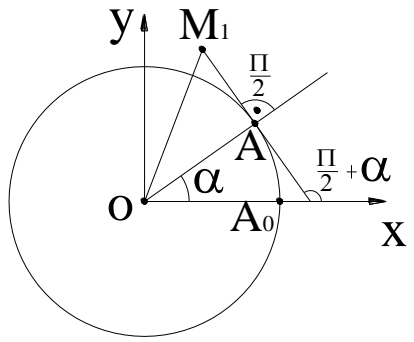

Figure 2: Geometry view

Projecting on the $\mathrm{x}$-axis we have:

$$
x=R \cos \propto+A M \cos \theta \cos \left(\left(\alpha+\frac{\pi}{2}\right)\right.
$$

$\rightarrow x=R \cos \propto-A M \cos \theta \sin \propto$

Projecting on the y-axis we have:

$$
y=R \sin \propto+A M \cos \theta \cos \left[\frac{\pi}{2}-\left(\propto+\frac{\pi}{2}\right)\right]
$$

$\rightarrow y=R \sin \propto+A M \cos \theta \cos \propto(2)$

Projecting on the z-axis we have:

$$
z=0+A M \cos \varphi
$$

$\rightarrow Z=A M \cos \varphi \quad(3)$

$\varphi$ is the angle between line $\mathrm{t}$ and $\mathrm{z}$-axis, $\theta$ is the angle between line $t$ and plane xoy,so the value of them are constant. We put $\cos \varphi=\mathrm{b}, \cos \theta=\mathrm{c}$ and $\mathrm{AM}=\mathrm{k} \alpha$ then we have the EQT of helix-curve of Hyos:

$x=R \cos \propto-k c \propto \sin \propto$

$y=R \sin \propto+k c \propto \cos \propto$

$z=k b \propto$

\subsection{Some special cases}

Case 1:

If the line 1 is parallel to the line $t$ then $\theta=90^{\circ}$.

Replace this value into equations (1), (2), (3):

$$
x=R \cos \propto
$$

$x=R \cos \propto$

$y=R \sin \propto$

$z=b \alpha$
This is an EQT of helix-sprail of a cylinder.

The EQT of the circle of it's:

$$
x^{2}+y^{2}=R^{2}
$$

Case 2

If line $t$ intersects line 1 then $\mathrm{OA}=\mathrm{R}=0$.

Replace this value into equations (1), (2), (3):

$x=-k c \propto \sin \propto$

$y=k c \propto \cos \propto$

$z=b \alpha(12)$

This is an EQT of helix-sprail of a cone.

The EQT of the circle of it is:

$x^{2}+y^{2}=R^{2}=k^{2} c^{2} \alpha^{2}$

$R=k c \propto 14)$

The property of a Hyos surface is its tangent to a fixed direction at a constant angle. We will prove this property.

Derivative of the equations (4), (5) and (6) we have:

$\dot{x}=-R \sin \propto-k c \dot{c} \propto \cos \propto-k \operatorname{csin} \propto$

$\dot{x}=-(R+k c) \sin \propto-k c \propto \cos \propto(15)$

$\dot{y}=R \cos \propto+k \cos \propto-k c \propto \sin \propto(16)$

$\dot{z}=k b(17)$

$\dot{x}^{2}=(R+k c)^{2} \sin ^{2} \propto+k^{2} c^{2} \propto^{2} \cos ^{2} \propto+$

$2(R+k c) k c \propto \sin \propto \cos \propto(18)$

$\dot{y}^{2}=(R+k c)^{2} \cos ^{2} \propto+k^{2} c^{2} \propto^{2} \sin ^{2} \propto-$

$2(R+k c) k c \propto \sin \propto \cos \propto(19)$

$\dot{z}^{2}=k^{2} b^{2}(20) \quad\left(\frac{d s}{d \alpha}\right)^{2}=\dot{x}^{2}+\dot{y}^{2} \dot{+} \dot{z}^{2}\left(\frac{d s}{d \propto}\right)^{2}=(R+k c)^{2}+$ $k^{2} c^{2} \propto^{2}+k^{2} b^{2}(21)$

$\frac{d s}{d \propto}=\sqrt{(R+k c)^{2}+k^{2} c^{2} \alpha^{2}+k^{2} b^{2}}(22)$

Define unit tangent vector:

$\vec{t}=\frac{\overrightarrow{d M}}{d \propto}=\overrightarrow{M_{1}}: \frac{d s}{d \propto}=\dot{x} \mathrm{i}+\dot{y} \mathbf{j}+\dot{z} \mathrm{n}$

Replace (15), (16), (17) into (23), we have: 
$\vec{t}=\frac{[-(R+k c) \sin \alpha-k c \propto \cos \propto\rfloor}{\sqrt{(R+k c)^{2}+k^{2} c^{2} \alpha^{2}+b^{2}}} \vec{l}+\frac{\mid(R+k c) \cos \propto-k c \propto \sin \propto\rfloor}{\sqrt{(R+k c)^{2}+k^{2} c^{2} \alpha^{2}+b^{2}}} \vec{\jmath}+$ $\frac{k b}{\sqrt{(R+k c)^{2}+k^{2} c^{2} \alpha^{2}+b^{2}}} \vec{n}$

$\vec{t} \sqrt{(R+k c)^{2}+k^{2} c^{2} \alpha^{2}+b^{2}} \quad=[-(R+k c) \sin \propto$ $-k c \alpha \cos \alpha i+R+k c \cos \alpha-k c \alpha \sin \alpha j+\mathrm{kb} n$

$\vec{h}=[-(R+k c) \sin \propto-k c \propto \cos \propto] \vec{i}+[(R+k c) \cos \propto$ $-k c \alpha \sin \alpha j+\mathrm{kb} n$

Vector $\mathrm{t}$ and vector $\mathrm{h}$ are the same direction.

$\mathrm{kb}$ is a constant, so the angle between vector $\mathrm{h}$ and the $\mathrm{z}-$ axis is a constant. Therefore, the angle between the vector $t$ and the $\mathrm{z}$ axis is also a constant.

Thus equations (4), (5) and (6) are general equations of helix-sprial of Hyos.

\section{PERPENDICULAR PROJECTIONS OF HYS}

\subsection{Top view}

The projection of a Hys on the XOY plane is:

$x=R \cos \propto-k c \propto \sin \propto$

$$
y=R \sin \propto+k c \propto \cos \propto
$$

The $\mathrm{x}$-axis is the symmetry axis of this curve.

A value of $x$ will give two value of $y$.

Fig 3 is the top view of Hys

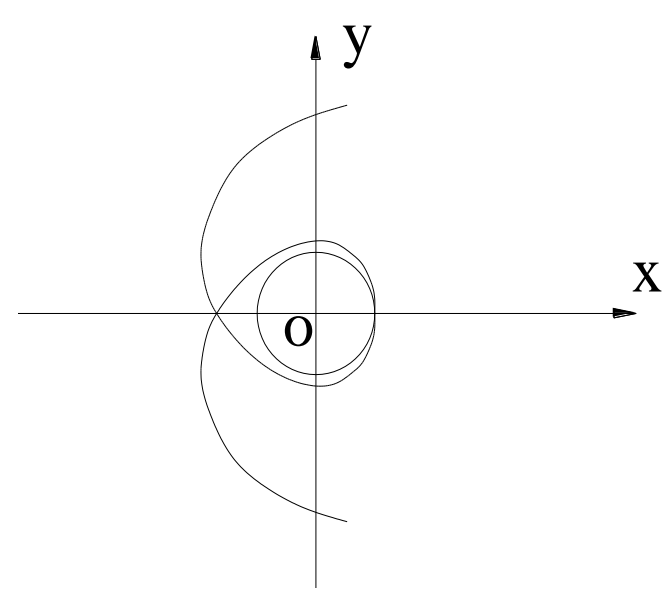

Figure 3: The top view of Hys

\subsection{Left view}

The projection of a Hys on the XOY plane is:

$y=R \sin \propto+k c \propto \cos \propto$

$$
z=k b \propto
$$

$y=R \sin \alpha+k \operatorname{cocos} \alpha=R\left(\sin \alpha+\frac{k \operatorname{co} \alpha}{R} \cos \alpha\right)$

We set:

$\frac{k c \alpha}{R}=\operatorname{tg} \varphi=\frac{\sin \varphi}{\cos \varphi}$

$y=R\left(\sin \alpha+\frac{\sin \varphi}{\cos \varphi} \cos \alpha\right)$

$\rightarrow y=\mathrm{R}\left(\frac{\sin \alpha \cos \varphi+\cos \alpha \sin \varphi}{\cos \varphi}\right)$

$\rightarrow y=\mathrm{R} \frac{\sin (\alpha+\varphi)}{\cos \varphi}$

$\cos \varphi=\frac{1}{\sqrt{1+\operatorname{tg}^{2} \varphi}}$

$\cos \varphi=\frac{1}{\sqrt{1+\frac{k^{2} c^{2} \alpha^{2}}{R^{2}}}}$

$\cos \varphi=\frac{R}{\sqrt{R^{2}+k^{2} c^{2} \alpha^{2}}}$

$y=\sin (\alpha+\varphi) \sqrt{R^{2}+k^{2} c^{2} \alpha^{2}}(29)$

This is the EQT for the sine function.

\subsection{Front view}

$x=R \cos \alpha-k \cos \sin \alpha=R\left(\cos \alpha-\frac{k c \alpha}{R} \sin \alpha\right)$

$\frac{k c \alpha}{R}=\operatorname{cotg} \gamma=\frac{\cos \gamma}{\sin \gamma}$

$x=R\left(\cos \alpha-\frac{\cos \gamma}{\sin \gamma} \sin \alpha\right)$

$\rightarrow x=\mathrm{R}\left(\frac{\sin \gamma \cos \alpha-\cos \gamma \sin \alpha}{\sin \gamma}\right)$

$\rightarrow x=\mathrm{R} \frac{\sin (-\alpha-\alpha)}{\sin \gamma}$ 


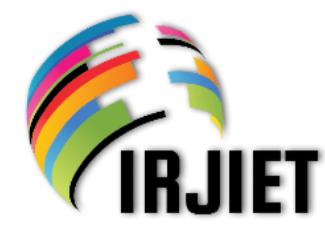

$\sin \gamma=\frac{1}{\sqrt{1+\operatorname{cotg}^{2} \gamma}}$

$\sin \gamma=\frac{1}{\sqrt{1+\frac{k^{2} c^{2} \alpha^{2}}{R^{2}}}}$

$\sin \gamma=\frac{R}{\sqrt{R^{2}+k^{2} c^{2} \alpha^{2}}}$

$x=\sin (y-\alpha) \sqrt{R^{2}+k^{2} c^{2} \alpha^{2}}$

This is the EQT for the sine function.

Since its axis is perpendicular to the plane (XOY), so its front view and left side views are the same.

\section{SOME APPLICATIONS OF HYOS AND HYS}

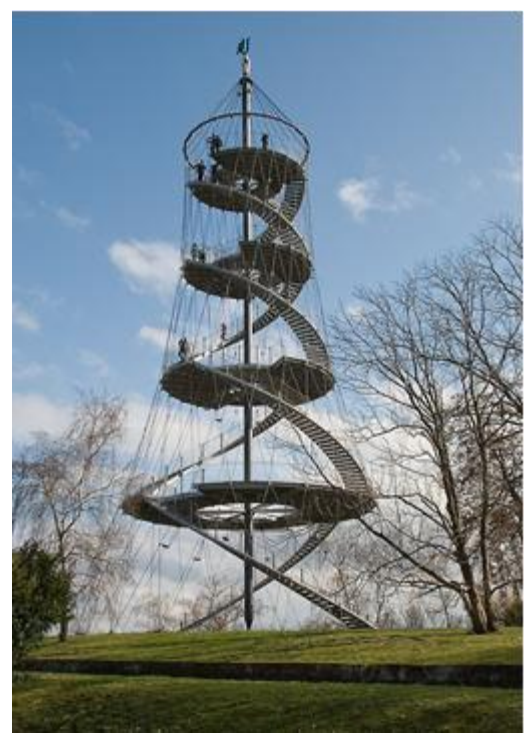

Figure 4: The Killesberg observation tower, Stuttgart, Germany, 2001

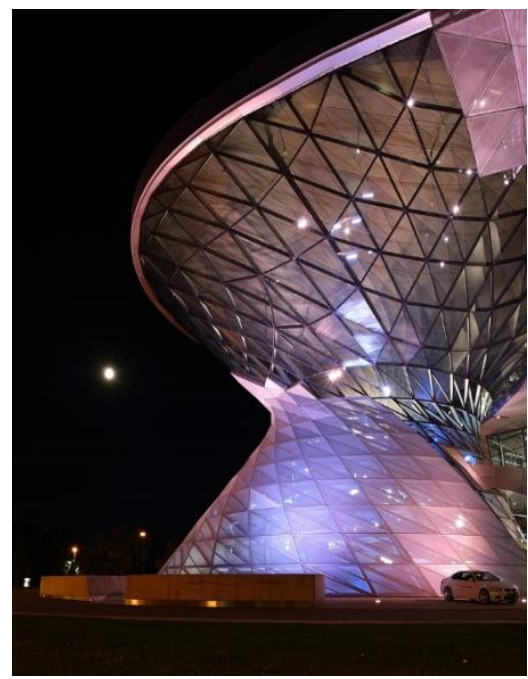

Figure 5: Museum and event vanue, Munich, Germany 2007
ISSN (online): 2581-3048

Volume 4, Issue 9, pp 1-5, September-2020 https://doi.org/10.47001/IRJIET/2020.409001

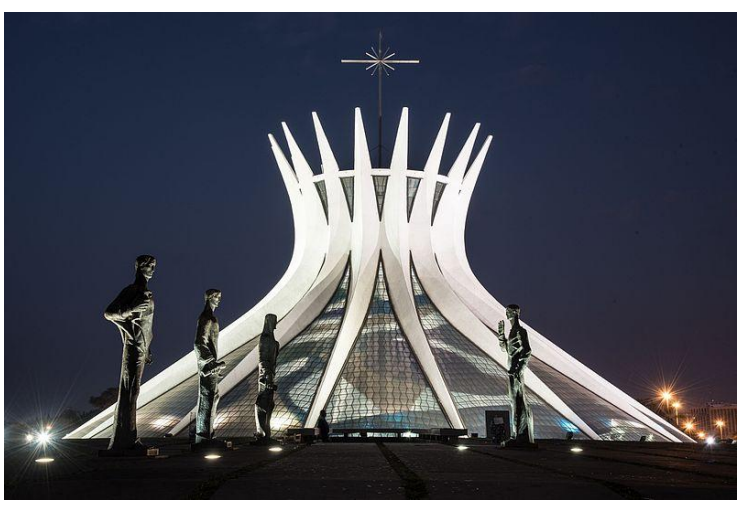

Figure 6: Cathedral of Brazil, 1970

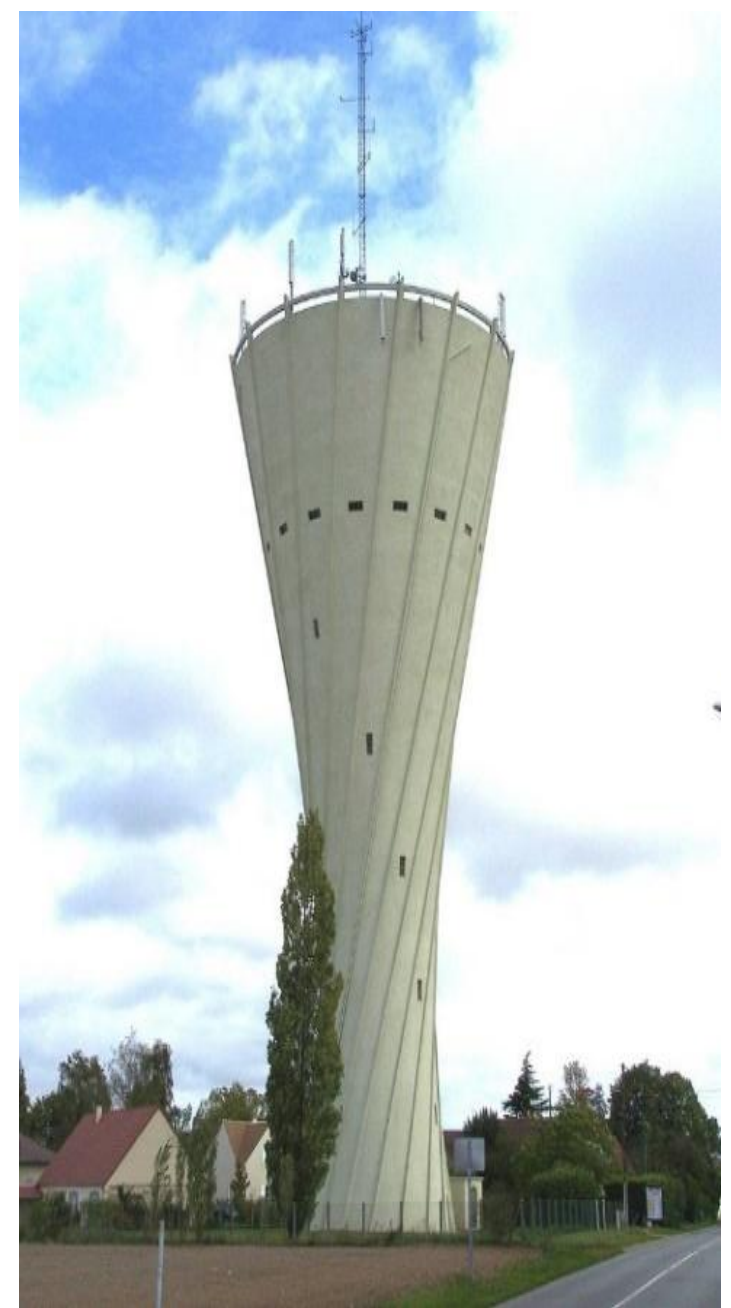

Fig 7: Essart-le -Roi, water tower of France

The Killesberg observation tower, Stuttgart, Germany, 2011 Hyperboloid single-sided hand

\section{CONCLUSION}

In mechanical engineering there are two cylindrical and conical spiral lines. These helical lines to form helical surfaces are called threads. This article has presented a new spiral. It is a Hys. It also applies to devices or towers with their surfaces are Hyos. Some pictures of its application are referenced above. 
ISSN (online): 2581-3048

Volume 4, Issue 9, pp 1-5, September-2020 https://doi.org/10.47001/IRJIET/2020.409001

\section{REFERENCES}

[1] Nguyen Dinh Tri (editor-in-chief), Advanced Mathematics, volume 1, Education Publishing House 2006. ... Nguyen Thuy Thanh, Background of complex variable theory, VNU Publishing House, 2006.

[2] Dinh Tri Nguyen (editor) - Van Dinh Ta - Ho Quynh Nguyen ... Advanced Math Exercises Episode 1 Dinh Tri Nguyen June 3, 2018In "Curriculum".

[3] Mechanics Program (Episode 2: Dynamics) - Sanh Do (Editor). September 2016 - publisher of Hanoi University of Science and Technology.

[4] https://en.wikipedia.org/wiki/Hyperboloid

\section{AUTHOR'S BIOGRAPHIES}

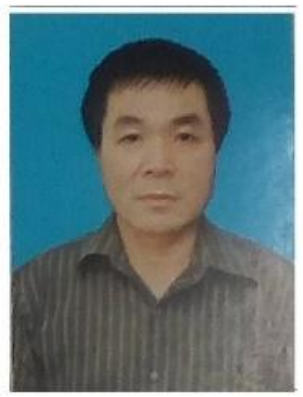

Manh Hong Do M. E (1968), Degrees in mechanical engineering from $\mathrm{Ha}$ Noi University of Science and technology. He is a Mater, Department of Mechanical Engineering, Ha Noi University of Science and Technology.

\section{Citation of this Article:}

Manh Hong Do, "Some Applications of Hyperboloid of One Sheet and How to Make the Equation of Helix-Spiral of It" Published in International Research Journal of Innovations in Engineering and Technology - IRJIET, Volume 4, Issue 9, pp 1-5, September 2020. https://doi.org/10.47001/IRJIET/2020.409001 